\title{
An inexpensive computer and interface for research in the behavioral sciences
}

\author{
RICHARD J. NICHOLLS and RANDALL M. POTTER \\ Clarion State College, Clarion, Pennsylvania 16214
}

\begin{abstract}
The Sinclair ZX81 computer and Byte-Back BB-1 control module are very inexpensive devices that can be used to control equipment in a psychology laboratory. A simple program and description for interfacing the system to a Skinner box are presented.
\end{abstract}

Sinclair Research Ltd. ${ }^{1}$ introduced a personal computer (the ZX81) that has received excellent reviews (e.g., Solomon, 1982) and is currently available for less than $\$ 100$ assembled $^{2}$ with a 1-KB RAM and Extended BASIC. Timex Corporation is marketing a similar 2-KB RAM machine as the "Timex Sinclair (TS) 1000" for the same price in stores where Timex products are sold (see Hawkins, 1982). Software reviews, descriptions of peripherals, and helpful user hints can be found in two publications designed specifically to support the Sinclair ZX81 and TS 1000 called Syntax and Sync. ${ }^{3}$ The ZX81 and TS 1000 connect to any standard television receiver for video output, but they also operate without being connected to a television. This makes the systems even more convenient and inexpensive for control uses; a single video monitor and a cassette recorder can be used to program and retrieve data for several machines.

The Byte-Back Company ${ }^{4}$ is marketing products for the ZX81 and TS 1000 including a BB-1 control module with eight inputs and outputs. The Byte-Back BB-1 plugs directly into the computer's expansion port and can easily be controlled using "PEEK" and "POKE" commands (see the sample program in Figure 1). The BB-1 control module sells for $\$ 69$ wired and tested, comes with an excellent manual, and for those unaccustomed to the complex wiring of integrated circuits or programming in machine code, provides an excellent alternative to O'Dell's (1981) Z80-based controller.

The BB-1 also works with the ZX80, although the program given here requires some modification to be used with the integer BASIC of the older machine. Even though the ZX80 was offered with a 4-KB BASIC and the ZX81 and TS 1000 use an 8-KB ROM-based BASIC, the RAM memory maps for the three systems remain compatible. RAM begins at Location 16384 decimal and ends at 17408 for 1-KB machines (ZX80/81), 18432 for

Authorship status is alphabetical, and requests for reprints may be directed to either author at the Department of Psychology, Clarion State College, Clarion, Pennsylvania 16214. This project was supported, in part, by awards to both authors from the Clarion State College Foundation. the TS 1000 , or 32768 for all machines with the optional 16-KB memory pack. As the BB-1 uses memory addresses $32764-32767$, its operation is unaffected by changes in the BASIC program, and is well beyond the range of memory likely to be used in control operations.

\section{EXAMPLE}

Controlling a Skinner box with either computer (ZX81 and TS 1000) and a BB-1 is very easily accomplished by connecting the lever and pellet feeder to the edge connector on the BB-1. Connect the lever's microswitch " $\mathrm{C}$ " to Input $22 \mathrm{~B}$, the "NC" to Input $4 \mathrm{~B}$, and the "NO" to Input 7B. Connect the pellet feeder ${ }^{5}$ through Outputs 2A and 2B. The BB-1 manual provides the information necessary to understand the electrical configuration of inputs and outputs.

The program shown in Figure 1 (which should be run in the FAST mode) produces a standard fixed-ratio schedule of reinforcement and records the time between reinforcements in seconds. This program is written so that no additional external pulse formers are needed to detect switch closures or to operate the pellet feeder. However, for those researchers expecting to measure very high rates of responding (e.g., pigeon pecking), we suggest that an external pulse former be used to operate the feeder and that Line 160 (PAUSE 3) be eliminated so as not to miss any responses by the subject.

The program allows recording of approximately 30 data points within the 1-KB limitation of the ZX81. Deletions of Lines 10,20,180, and 210 and alteration of Line 190 to print only the value of T (i.e., 190 PRINT T;“" ";) allows recording of well over 100 values. Sample runs of the program produce time values consistent with an external $.01-\mathrm{sec}$ stopclock $(\mathrm{r}>.99)$. For researchers demanding even more precise control over timing functions, Sinclair Research, Ltd., recently announced their newest computer, the Sinclair ZX Spectrum (see Rodwell, 1982). Although not yet available in the United States, the Spectrum is reported to have a real-time clock, color graphics, and 16-KB internal RAM for approximately $\$ 225$ ( $\$ 310$ for $48-K B$ internal RAM). It remains to be seen if the BB-1 will be com- 


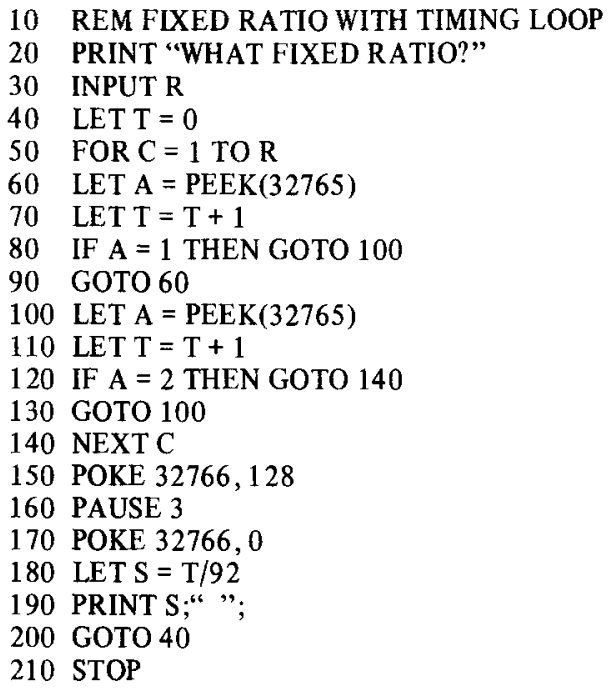

\author{
Remarks \\ Input F Rumber \\ Looks for NC switch \\ Looks for NO switch \\ Operates feeder \\ Turns feeder off \\ Computes seconds \\ Stores time in display file
}

Figure 1.

patible with the new Spectrum. However, the less expensive ZX81 and TS 1000 meet many research needs quite adequately.

Other schedules of reinforcement are easily programmed into the ZX81, and with the addition of the 16-KB memory (available for less than $\$ 50$ ), there is ample capability for summary analysis programs. As the array-handling capabilities of the computer are fairly broad (including multidimensional arrays), the extra memory is a useful addition for studies in which large amounts of data are generated.

\section{CONCLUSIONS}

The ZX81/TS 1000 combined with the Byte-Back BB-1 control module offer an inexpensive, yet reliable control system for laboratory research. Our recommendation for anyone interested in using the system is to purchase the 16-KB RAM pack, a cassette tape recorder for saving and loading programs, and a television monitor. Even with these additional purchases, the entire system can be obtained for approximately $\$ 325$. The system offers an attractive, low-cost alternative to other microprocessor-based control systems currently available.

\section{REFERENCES}

Hawkins, W. J. Now: World's first $\$ 100$ personal computer. Popuiar Science, August 1982, pp. 54-56.

O'DeLl, J. W. The MicroAce: An inexpensive computer controller. Behavior Research Methods \& Instrumentation, 1981, 13, 682-685.

Rodwell, P. Sinclair ZX spectrum. Popular Computing, September 1982, pp. 56-61.

Solomon, L. Sinclair ZX81 personal computer. Popular Electronics, March 1982, pp. 32; 34.

\section{NOTES}

1. Sinclair Research, Ltd., 2 Sinclair Plaza, Nashua, New Hampshire 03061.

2. See ad in Creative Computing, July 1982, pp. 31-33.

3. Syntax is published by The Harvard Group, R.D. No. 2, Box 457, Harvard, Massachusetts 01451 , and subscriptions for Sync are available at 39 East Hanover Avenue, Morris Plains, New Jersey 07950.

4. Byte-Back Company, Route 3, Box 147, Brodie Road, Leesville, South Carolina 29070.

5 . To prolong the life of the contacts in the BB-1's relays, we suggest using a slave relay to drive the pellet feeder.

(Received for publication July 23, 1982; revision accepted October 12, 1982.) 